\title{
The processing of spatially transformed text
}

\author{
KEITH D. HORTON \\ Wilfrid Laurier University, Waterloo, Ontario, Canada
}

\begin{abstract}
The present study shows that previous attempts to demonstrate explicit memory for orientation of spatially transformed text were inconclusive. In the present experiments, subjects read spatially transformed sentences and were then given a recognition test that permitted the use of only nonsemantic information. The results revealed clear evidence of incidental retention of nonsemantic information on an immediate test, no improvement in performance under intentional learning conditions, reduced but still better than chance retention following a 48-h delay, and little change in performance with anomalous sentences. These and other results involving the reading of spatially transformed materials are discussed in terms of the interaction of processing requirements at encoding and retrieval.
\end{abstract}

Research on human memory has demonstrated that, under most circumstances, information is better remembered when attention has been directed to the semantic, rather than the nonsemantic, features of the stimuli (e.g., Craik \& Tulving, 1975). However, there is substantial evidence that nonsemantic features are well remembered under a wide variety of experimental conditions. Hunt and Elliott (1980) and Kolers and Roediger (1984) reviewed some of this evidence. One of the primary sources of evidence cited by these and other authors in support of this conclusion has been research reported by Kolers and his colleagues in which subjects were asked to read material presented in one of several spatial transformations (see Kolers, 1979; Kolers \& Roediger, 1984). The purpose of the present paper is, first, to demonstrate that the results of these studies are inconclusive due to problems of design and analysis, and then to describe some research that provides a clear test of memory for nonsemantic features (namely, spatial orientation) within this paradigm. It seems necessary to provide such a test because data from this paradigm are very commonly cited as evidence for the memory of nonsemantic information.

Subjects in Kolers's experiments were typically asked to read paragraphs presented in one of the several spatial orientations (see Kolers, 1979, for a review). In some cases, the material was presented in normal upright orientation, whereas in others, it was presented inverted, reversed from right to left, or in some other spatial transformation. The logic for using this task to assess the memorability of nonsemantic information rests on the assumption that spatial transformation of the material changes the perceptual or graphemic features of the stimuli but has no effect on the semantic features. Thus, Kolers $(1979$, p. 366) assumed that sentences presented in different orientations "are equivalent as semantic objects."

This research was supported by Natural Sciences and Engineering Research Council of Canada Grant A7454 to the author. Requests for reprints should be addressed to Keith D. Horton, Department of Psychology, Wilfrid Laurier University, Waterloo, Ontario N2L 3C5, Canada.
Consequently, Kolers and his colleagues viewed the superior retention of transformed material over normally oriented material as evidence for the role of elaborated graphemic information in memory.

In terms of methodology, these studies may be conveniently grouped into "read-and-sort" studies and "rereading-time" studies. The latter group includes those in which subjects read each of several passages more than once (Kolers, 1975b, 1976). The principal finding from these rereading-time studies is that sentences presented in a transformed orientation on the second presentation are reread faster when they were originally read in that orientation than when they were originally read in normal orientation. Kolers (1979) concluded that the faster rereading times reflected the enhanced graphemic information available from the prior reading of the sentence.

Other researchers have presented evidence that suggests that the rereading-time benefits may be the result of enhanced semantic, rather than graphemic, information stored in memory (Graf, 1981; Horton, 1985; Masson \& Sala, 1978; Tardif \& Craik, 1989). For example, Masson and Sala (1978, Experiment 2) found equivalent rereading times for verbatim and paraphrased sentences when the sentences were read in a transformed orientation on both presentations. In a similar vein, Horton (1985) found that the rereading times for sentences presented twice in the same transformed orientation were no faster than those for sentences presented twice in different transformed orientations. Since the graphemic information available from the first reading of a sentence would be of little or no use when rereading the sentence either with a different wording (Masson \& Sala, 1978) or in a different spatial transformation (Horton, 1985), these findings appear to demand an interpretation based on memory for other than graphemic features.

The data from these more recent studies indicate that the rereading-time measure is unaffected by detailed graphemic information remembered from the prior reading of the sentence. The question remains, however, whether the benefits of the additional graphemic process- 
ing that seems to be necessary for reading spatially transformed text can be observed on a memory test that directly taps this information.

The read-and-sort task (Kolers, 1973, 1974, 1975a; Kolers \& Ostry, 1974) has provided the most commonly cited evidence on this question. In this task, subjects are presented with a set of sentences (the read deck), which they are asked to read as quickly and as accurately as possible. A second deck (the recognize deck) is then presented, which includes some of the sentences that appeared previously in the read deck ("old") along with other sentences not previously read ("new"). Furthermore, some of the old sentences are presented in exactly the same orientation (same form) in which they appeared in the read deck, whereas others are presented in a different orientation (different form). The subject's task is to identify each sentence of the recognize deck as old or new, and, if old, as in the same form or a different form. On the basis of a series of signal detection analyses conducted on the sorting of the recognize deck, Kolers (e.g., 1979) concluded that subjects demonstrated good retention of graphemic information for sentences originally presented in other than normal orientation. Some comments on these analyses are in order, inasmuch as they provide some of the primary support for Kolers's theoretical position.

Three values of $d^{\prime}$ are of interest. For $d^{\prime}(\mathrm{sem})$, hits are defined as old sentences classified as old, and false alarms are defined as new sentences classified as old. This measure is used as an index for the recognition of a sentence "as a semantic object" (Kolers, 1979, p. 367). For $d$ '(new), hits are old sentences placed in the correct pile (same form, different form) and false alarms are new sentences placed in the respective piles (same form, different form). This measure is used as an index of memory for graphemic information. Finally, for $d^{\prime}$ (old), hits are defined as for $d^{\prime}$ (new), and false alarms as old sentences misplaced in the same form or different form piles. This measure indexes "memory of the sentences as pictorial objects”' (Kolers, 1979, p. 367).

Kolers (e.g., 1974, 1979) interpreted $d^{\prime}(\mathrm{sem})$ as reflecting the semantic information available about a sentence from its original encoding in the read deck. It is not clear, however, that simply identifying a sentence as old or new necessarily involves strictly semantic information, to the exclusion of other types of information. Indeed, there is some suggestion that $d^{\prime}(\mathrm{sem})$ is not entirely independent of $d^{\prime}$ (old) (e.g., Kolers, 1974), and that recognition of a sentence can be influenced by memory of perceptual features (Masson, 1984). Consistent with these findings are other data showing that performance on a recognition test may be sensitive to a variety of components (e.g., Johnston, Dark, \& Jacoby, 1985; Mandler, 1980). Thus, its label notwithstanding, there is some doubt as to whether the $d^{\prime}(\mathrm{sem})$ measure actually indexes the use of semantic information.

Also of interest is the reasoning by which $d^{\prime}$ (new) [or $d^{\prime}$ (typog), as Kolers, 1979, would prefer] is said to reflect the retention and use of graphemic or typographic infor- mation, whereas $d^{\prime}$ (old) is said to reflect the use of pictorial information. It seems at least as legitimate to suggest that the ability to correctly discriminate old sentences as appearing in the same form or a different form [i.e., the $d^{\prime}$ (old) measure] reflects memory for graphemic information, as does the $d^{\prime}($ new) measure (Masson \& Sala, 1978). Furthermore, it does not seem appropriate to assess memory for graphemic information by a comparison with new sentences given as false alarms, since such errors may relate to the use of a variety of types of information, of which graphemic information is just one. These arguments suggest that perhaps $d^{\prime}$ (old) is an alternative index of memory for graphemic information. ${ }^{1}$

Given this perspective, it is of interest to note that the $d^{\prime}$ (old) values are generally smaller than the $d^{\prime}$ (new) vallues (e.g., Kolers, 1973, 1974), and, indeed, are only marginally different from zero with other than an immediate test (Kolers \& Ostry, 1974). Viewed in this way, the data from the read-and-sort studies do not provide particularly strong evidence of memory for graphemic information.

In addition to these issues concerning the interpretation of the various $d^{\prime}$ measures, a second problem arises in the interpretation of the data from the read-and-sort studies. In most of these studies (e.g., Kolers \& Ostry, 1974; see also Masson, 1984), only the normal and inverted orientations are used for presentation of sentences in the read deck. As noted previously, evidence from the more recent rereading-time studies (Graf, 1981; Horton, 1985; Masson \& Sala, 1978) has strongly supported the conclusion that extensive semantic processing is carried out on spatially transformed sentences, relative to normally oriented sentences. Accordingly, it seems possible that subjects' sorting of the recognize deck in the readand-sort studies could be based on the amount or quality of information remembered about each sentence: The more information available in memory, the more likely it is that the sentence was originally read in the transformed orientation. Although the necessary supporting data have not been reported, this interpretation requires only that subjects have the metamemorial skills to recognize that the more difficult task of reading spatially transformed material results in an enriched memory trace. Since this enriched memory trace may include graphemic and/or semantic components, it is evident that such studies are unable to isolate the role of graphemic information in sorting performance.

One study reported by Kolers (1974) does not suffer from this interpretive problem. His subjects read sentences in normal orientation and in two different transformed orientations. However, the data were analyzed using the $d^{\prime}$ measures described above, and thus their interpretation is subject to the earlier concerns regarding these measures. Nevertheless, the raw data on hit and false-alarm rates are useful in reconsidering the findings. If we deal first with those sentences which were presented in the same orientation in the read and recognize decks (NN, II, rRrR; see Note 1 for explanation), we find that the 
proportion of sentences that were recognized as old and were assigned to the same form pile was not markedly higher for the two transformed orientations $(I I=.708$, $\mathrm{rRrR}=.806$ ) than for the normally oriented sentences $(\mathrm{NN}=.739)$. A similar pattern occurs for sentences appearing in two different orientations in the read and recognize decks. Those originally read in the normal orientation were correctly identified as being in a different form in the recognize deck when tested in a transformed orientation ( $\mathrm{NI}=.833, \mathrm{NrR}=.762)$ approximately as often as were those originally presented transformed and tested transformed $(\mathrm{IR}=.763, \mathrm{rRI}=.867)$. These data provide no evidence that subjects remember the graphemic details of spatially transformed materials any better than those of normally oriented materials.

The present experiments were designed to provide more direct tests of the memorability of graphemic information following the reading of spatially transformed materials. The procedures and measures were selected to eliminate the difficulties in interpretation of the earlier data. In these experiments, the subjects read sentences, each presented in one of three possible spatial transformations. Later, the subjects were presented with a threealternative forced-choice test in which each of the sentences was shown in all three possible orientations. The subjects' task was to select the orientation in which they had previously read the sentence. Since there is no reason to expect that subjects' processing of semantic information will vary as a function of a sentence's appearing in, say, inverted or reversed orientation, it seems clear that this test can only be performed using graphemic information. If the specific graphemic processing associated with a given transformation is available, subjects will be able to select the correct version of the sentence with better than chance accuracy.

\section{EXPERIMENT 1}

Experiment 1 was designed with two specific purposes in mind. The first was to determine the memorability of spatial orientation under incidental learning conditions. Although the materials and procedures of other relevant studies vary widely, research has shown that memory for perceptual and graphemic details can be at or near chance in some situations but reliably above chance in others. For example, Graesser and Mandler (1975) found that subjects who performed a semantic orienting task on sentences were later unable to discriminate the target sentences from sentences that differed from the targets in terms of surface structure but not meaning. Similarly, Bransford and Franks (1971) reported that subjects were unable to discriminate target sentences from sentences containing different combinations of the idea units of previously seen sentences. By contrast, Geiselman and Bellezza (1976) and Geiselman and Bjork (1980, Experiment 2) reported that the sex of the speaker of a sentence was remembered reliably better than chance, although performance on their task may have been affected by semantic processing factors (Geiselman \& Bellezza, 1977).

The second purpose of this experiment was to determine whether memory for the spatial orientation of sentences would be enhanced if subjects were explicitly instructed about the nature of the memory test. Frost (1972, Experiment 2) found that subjects who expected to be tested for physical details of stimulus items tended to cluster recalled items more on the basis of their physical features than did subjects who expected to be tested for free recall of the names of the items. In addition, a subsequent recognition test revealed better memory for physical details by subjects who expected to be tested for these features. Also, Graesser and Mandler (1975, Experiment 2) found that, compared with subjects instructed to attend to the meaningfulness of sentences, subjects instructed to attend to "grammaticality" were subsequently much better at discriminating targets from distractors differing only in surface structure. Finally, although no incidental learning condition is available for comparison purposes, Yekovich and Thorndyke (1981) reported that subjects were reliably able to discriminate target sentences from paraphrases of targets when given intentional learning instructions. These data appear to suggest that attention to perceptual features, either through intentional learning instructions or through an orienting task that focuses on these features, enhances the memorability of these features.

Geiselman and Bjork (1980) provided one apparent exception to this conclusion. They found that subjects who "rehearsed" target items in the voice of the speaker were, on a subsequent test, no more able to identify the voice that spoke a particular target item than were subjects who generated associations to target items in the voice of the speaker. It is possible, however, that subsequent identification of the voice of the target items in the latter condition may have been mediated by memory for the "voice" of the generated associations. In sum, then, it appears that attention to perceptual features of stimuli may increase memory for these features.

In the present experiment, the subjects were presented with sentences in each of three different spatial transformations. Immediately after reading the sentences, the subjects were given a recognition test in which they were asked to select the orientation in which each sentence had appeared previously. One group was given incidental learning instructions and one was given intentional learning instructions for the orientation of the sentences. It was predicted that both groups would show better than chance accuracy on a memory test for orientation, and also that the intentional group would show better memory than the incidental group. The first prediction is consistent with Kolers's hypothesis that perceptual features form a critical part of the processing of information, particularly when the perceptual features are novel or "disparate" (Kolers \& Roediger, 1984). 


\section{Method}

Subjects. Twenty-four undergraduate volunteers were tested individually. The data from 7 additional subjects were discarded, 3 due to experimenter error and 4 for making too many reading errors within any cell of the design.

Design and Materials. The design was a 3 (orientation) $\times 2$ (instruction) mixed factorial, with repeated measures on the former variable. Sixty semantically related sentences were used in the experiment. These materiais are described more completely elsewhere (Horton, 1985), but, briefly, these sentences were liberal adaptations of every fourth sentence from an undergraduate social psychology text (Aronson, 1976). The sentences were always presented in the same sequence as in the original source so as to maintain the semantic theme.

All subjects initially read the first 12 sentences from the larger set as practice sentences. The three spatial orientations were used equally often within the practice set. These sentences were not tested, but some were used to illustrate the nature of the memory test.

The 48 experimental sentences were divided into two sets by assigning sentences to sets alternately, based on the order of the sentences in the original source. In addition, 3 sentences from each set were also presented in the other set. Thus, a total of 6 sentences appeared in both sets (see Horton, 1985). Twelve subjects were tested on each of the sets. Although the order of sentences within each set did not change, each sentence was presented in each orientation equally often over the entire experiment. Input positions were divided into three blocks of nine, within which each orientation $\alpha$ curred equally often.

In the test booklet, each of the 27 experimental sentences in a set was presented three times on a single page. The three orientations of each sentence were randomly ordered vertically on the page, and the pages themselves were randomly ordered for each subject. A space was provided to the left of each sentence for the subject to place a check mark. The subjects were also asked to indicate their degree of confidence in the selected orientation. A 5-point rating scale appeared at the bottom of each test page, along with a coding scheme that indicated that 1 signified strictly a guess and 5 signified very confident.

The inverted (I) orientation involved a mirror image of the sentence on the $x$-axis; the reversed (R) orientation involved a mirror image on the $y$-axis; the combined (C) orientation involved a mirror image on both the $x$ - and $y$-axes. It should be noted that the $I$ and $R$ transformations are not identical to those designated $I$ and $R$ by Kolers (1979, p. 365).

Each sentence was presented as a three- to five-line display, typed in 10-pitch Dual Gothic font. A small arrowhead identified the beginning of each sentence. The sentences were filmed and mounted on slides for projection on a screen.

Procedure. The subjects were told that their task was to read everyday English sentences presented in other than normal orientation. Each orientation was carefully illustrated through the use of examples. The instructions indicated that the sentences were to be read aloud as quickly and accurately as possible. If the subjects detected a reading error prior to completing the sentence, they were to return to the error, correct it, and then continue. The subjects were told that their total reading time was taken from the initial onset of the sentence until it was completed. Timing commenced automatically with stimulus onset and terminated when the experimenter pressed a button in response to correct reading of the final word in the sentence. This response by the experimenter also terminated presentation of the slide and initiated a 3-sec blank interval during which a slide change occurred and the reading time was recorded. Subjects in the incidental learning condition were given no information about the recognition test that was to follow. Subjects in the intentional learning condition were told that they would receive a recognition test in which each sentence would appear in each of the three possible orientations, and that their task would be to select the orientation in which they had previously seen the sentence. Accordingly, they were encouraged to attempt to deliberately remember the orientation of each sentence as it was read.

The recognition test began immediately following completion of the reading task. The subjects were fully informed, through the use of examples, as to the nature of the test. They were told that each page of the test booklet contained three presentations of a sentence previously read. Their task was to place a check beside the orientation in which the sentence had been read previously. After selecting one of the three orientations, they were also to provide a confidence rating by circling the appropriate number on the rating scale at the bottom of each page.

\section{Results}

An alpha level of .05 is used throughout this paper as the criterion for statistical significance.

Subjects were replaced if they made reading errors during the study trial on four or more of the nine sentences presented in any of the three orientations. Subsequent analyses of just those subjects who satisfied this criterion indicated that performance on the recognition test was unaffected by whether the few remaining sentences for which there were reading errors were included in or excluded from the data. Therefore, the data analyses based on all sentences read by these subjects are reported here.

Reading times. Analysis of median reading times revealed a significant effect of orientation $[F(2,44)=67.71$, $\left.M S_{\mathrm{e}}=54.03\right]$, but no effects associated with the instruction variable. A Fisher's test ( $1 \mathrm{sd}=4.44)$ indicated no differences in reading times for the $I(38.33 \mathrm{sec})$ and $R$ $(36.84 \mathrm{sec})$ sentences, but reading times for both were substantially longer than those for sentences with the $\mathrm{C}$ orientation $(16.24 \mathrm{sec})$. The $\mathrm{C}$ orientation requires more than twice the reading time of normally oriented sentences, as evidenced by other experiments in our lab using these materials. The mean error rates on the reading task were $8.33 \%, 5.56 \%$, and $0 \%$ for the $I, R$, and $C$ orientations, respectively.

Recognition test. The subjects were asked to select the orientation in which each previously read sentence had been presented. The recognition accuracy data appear in Table $I$ as a function of instruction condition and study orientation. Analysis of variance on the proportions of correct responses revealed that the only reliable effect was due to orientation $\left[F(2,44)=4.75, M S_{\mathrm{e}}=0.043\right]$. A Fisher's test (lsd $=0.126)$ indicated that the only reliable differences in performance were between the I and $R$ orientations. An important point to note from this initial analysis of the recognition data is that the instruction had no effect on performance $(F<1)$.

Further analyses of the recognition accuracy data indicated that the overall recognition performance for each of the three orientations was reliably better than chance (chance accuracy $=.33$ ) $[\operatorname{ts}(23)=11.58,4.49$, and 9.52 for the I, R, and C orientations, respectively].

The ease with which subjects read sentences presented in the $\mathrm{C}$ orientation suggests the possibility that the recognition data for this condition may suffer from the same confounding described earlier in the context of several of Kolers's studies: When subjects remembered a given sentence very well, they had only to choose between the 
Table 1

Recognition Test Performance as a Function of Instruction Condition (Experiment 1), Study Orientation, and Orientation Selected at Test

\begin{tabular}{|c|c|c|c|}
\hline \multirow{2}{*}{$\begin{array}{c}\text { Study } \\
\text { Orientation }\end{array}$} & \multicolumn{3}{|c|}{ Orientation Selected at Test } \\
\hline & 1 & $\mathbf{R}$ & $\mathrm{C}$ \\
\hline \multicolumn{4}{|c|}{ Experiment 1: Incidental Instruction } \\
\hline I & .806 & .065 & .130 \\
\hline $\mathbf{R}$ & .260 & .602 & .139 \\
\hline $\mathrm{C}$ & .213 & .083 & .704 \\
\hline \multicolumn{4}{|c|}{ Experiment 1: Intentional Instruction } \\
\hline I & .750 & .074 & .176 \\
\hline $\mathbf{R}$ & .204 & .583 & .213 \\
\hline $\mathrm{C}$ & .167 & .185 & .649 \\
\hline \multicolumn{4}{|c|}{ Experiment 2} \\
\hline I & .602 & .167 & .232 \\
\hline $\mathbf{R}$ & .380 & .389 & .232 \\
\hline $\mathrm{C}$ & .296 & .167 & .537 \\
\hline \multicolumn{4}{|c|}{ Experiment 3} \\
\hline I & .761 & .117 & .121 \\
\hline $\mathbf{R}$ & .194 & .597 & .208 \\
\hline C & .227 & .194 & .579 \\
\hline
\end{tabular}

Note $-\mathrm{I}=$ inverted, $\mathrm{R}=$ reversed, $\mathrm{C}=$ combined (see text).

$\mathrm{I}$ and $\mathrm{R}$ orientations on the recognition test, since these required the most extensive analyses during the reading phase. When very little information was retained, a sentence would be identified as having occurred in the $\mathrm{C}$ orientation. Effectively, this could mean that only the I and $\mathrm{R}$ sentences would be useful for assessing the memorability of graphemic information. The data argue against this hypothesis. To illustrate, consider sentences initially read in the I orientation. If subjects remembered enough about the processing of a sentence to determine that it was studied in either the I or the R orientation, but they did not have sufficient graphemic information to allow them to discriminate between the two, it would be expected that the proportion of I sentences identified as having occurred in the I and R orientations would be approximately equal. This was not the case: the proportions were, respectively, .778 and .069 , averaged across the incidental and intentional conditions (see Table 1). Furthermore, it would be expected that I sentences would be misidentified more frequently as $R$ than as $C$ sentences. Again, this was not the case: the proportions were, respectively, .069 and .153. Finally, performance on $C$ sentences could be expected to be markedly superior to that on both I and R sentences. Once again, this was not the case (see Table 1). Thus, the data strongly suggest that orientation is remembered quite accurately, regardless of how much additional graphemic processing may be required by the particular spatial transformation.

Confidence ratings were also provided on the recognition test. Individual subject medians were calculated, where possible. (In several cases, individual subjects made no errors in one or more cells of the design.) The confidence rating data appear in Table 2. Much of the variability evident in those cells that represent recognition er- rors may be attributed to many individual subject medians' being based on very few scores and, in some cases, the small number of subjects contributing to the cell.

Because of missing data for many subjects in one or more cells representing recognition errors, analysis of variance was carried out on confidence ratings assigned to correct responses only. The analysis revealed no reliable effects due to instruction condition, orientation, or their interaction (all $p \mathrm{~s}>.05$ ).

\section{Discussion}

This experiment was designed to assess the memorability of graphemic information, unconfounded by memory for other stimulus features, and also to assess the effects of explicit instructions to remember graphemic information. The first key finding is that memory for all three orientations was significantly above chance. Although perceptual or graphemic features of stimuli may not be remembered accurately under all conditions (Bransford \& Franks, 1971; Graesser \& Mandler, 1975), the present data clearly suggest that the transformed spatial orientation of previously read sentences is one such feature that is remembered significantly better than chance. This finding is consistent with Kolers's (1979) hypothesis that spatial transformation of a sentence demands enhanced processing of the graphemic details of the display, thereby resulting in the retention of more information about the stimulus features. This finding is particularly notable in the context of previous findings (Horton, 1985) that memory for spatial orientation had no measurable ef fect on the rereading time for a sentence. Thus, the relevant information about spatial orientation of a previously read sentence is available to the subject when the sentence is reread, but either is not used to facilitate rereading or is ineffective. Observation of the subjects performing the recognition test in the present experiments indicated that the subjects found the test very demanding, suggesting that the orientation of the sentence on its previous presentation is not quickly or readily brought to mind. Under these circumstances, subjects rereading a sentence as quickly as possible would not be expected to allocate processing resources to assess this information. Alternatively, of course, subjects may attempt to make use of this information while performing the rereading task, but find it to be ineffectual. Neither the procedures of the previous research nor those reported here allow us to discriminate among these possibilities.

Experiment 1 was also designed to assess the effects of intentional learning instructions on memory for spatial orientation. In contrast to evidence from other studies, cited earlier, intent to learn had no effect on memory performance. There are several possible explanations for this outcome. First, the lack of a statistical difference between the incidental and intentional learning groups could be attributed to the relatively small number of subjects assigned to each group. However, the issue of statistical power would seem to be obviated by the finding that the intentional group actually showed poorer memory for spatial 
orientation than did the incidental group. It could also be argued that the instructions to remember spatial orientation were not sufficiently strong to induce the subjects to engage in any additional processing that might enhance their memory for this information (see Postman \& Kruesi, 1977). However, although it is difficult to offer a firm conclusion regarding this interpretation, the instructions made explicit reference to the fact that each sentence would appear in all three possible orientations and that the task would be to select the orientation in which the sentence had been read previously. The instructions clearly emphasized the importance of paying attention to the orientation during the reading task.

Two other interpretations seem somewhat more plausible. First, perhaps the subjects did not know how to modify or elaborate their processing of the sentences in order to improve their memory for spatial orientation. It seems possible to address this hypothesis by giving the subjects an appropriate orienting task (Graesser \& Mandler, 1975) or several study/test sequences during which they might be able to develop an appropriate encoding strategy. Second, it may be that the reading task did not allow the subjects the opportunity to complete additional graphemic processing for purposes of the recognition test. Because the subjects were asked to read each sentence as quickly as possible and then, immediately upon completion of the reading task, the sentence was removed from the screen, any potential benefits of intentional learning instructions may have been eliminated simply due to lack of opportunity. Although the present experiment was not designed to address these alternatives, it seems possible that either or both of these factors may have been important in the finding of no memorial enhancement from the intentional learning instructions.

\section{EXPERIMENT 2}

One of the notable features of Kolers's work using the task of reading spatially transformed text is his conclusion that information about graphemic details may be retained over unexpectedly long delays (Kolers, 1976; Kolers \& Ostry, 1974). This conclusion is based on the procedures and analyses discussed earlier. Specifically, in one case in which the critical test trials were carried out more than a year after the initial reading, Kolers (1976) reported that previously read sentences were reread faster than sentences not previously read (Experiment 1). Also, there was limited evidence that at least some subjects may have been able to identify explicitly that a sentence had been read many months previously (Experiment 2). Neither finding, however, can be unequivocally attributed to memory for graphemic information. The data from the first experiment are based on sentences read in the same transformed orientation on the two presentations, and thus the reading speed benefits could as readily be attributed to memory for semantic information (Horton, 1985). The data from the second experiment are based strictly on an old/new discrimination task, which also need not reflect memory for graphemic features.

Kolers and Ostry (1974) utilized the read-and-sort task, with the sorting task on the recognize deck occurring as long as 32 days after the initial reading task. As noted earlier, only the $\mathrm{I}$ and $\mathrm{N}$ orientations were used in this study (see also Masson, 1984), thereby permitting interpretations based on memory for other than graphemic information. Also, the signal detection analyses are subject to the interpretive problems noted earlier.

Research using other paradigms has provided some suggestion that subjects may be able to remember graphemic information over at least a few days. For example, Glisky and Rabinowitz (1985) demonstrated that subjects could remember the specific form of a stimulus presented $48 \mathrm{~h}$ earlier. The subjects were initially asked to generate an item based on a work fragment cue in which two letters were missing. Two days later, they were able to identify the word fragment among other fragments of the same word at a better than chance level of accuracy. These data suggest that nonsemantic information extracted from a single stimulus event may be very durable in memory.

The present experiment was designed to determine whether sentence orientation is remembered over a $48-\mathrm{h}$ delay. Except for the longer test delay, Experiment 2 was very similar to Experiment 1.

\section{Method}

Twelve subjects were tested over two sessions, $48 \mathrm{~h}$ apart. The data from 4 additional subjects were discarded, 2 because they made four or more reading errors within any cell during the first session and 2 because they failed to return for the second session. During the first session, the subjects were told that they would be performing a series of tasks over the two sessions so that we could correlate performance on the various measures. Two tasks were completed during the first session. The subjects began by performing the reading task and then were asked to generate names of several states, based on word fragments; this was followed by a free-recall test of all $\mathbf{5 0}$ states. The second session included only the unexpected recognition test for sentence orientation. In all other regards, the design, materials, and procedure were identical to those of Experiment 1.

\section{Results}

Reading times. Analysis of median reading times revealed a reliable effect of orientation $[F(2,22)=23.31$, $\left.M S_{\mathrm{e}}=91.21\right]$. A Fisher's test $(\mathrm{lsd}=8.07)$ indicated that all pairwise comparisons differed reliably. The median reading times were $44.21,35.16$, and $18.00 \mathrm{sec}$ for the $\mathrm{I}, \mathrm{R}$, and $\mathrm{C}$ orientations, respectively. The mean error rates were $16.67 \%, 18.06 \%$, and $5.56 \%$ for the I, R, and C orientations, respectively.

Recognition test. The recognition accuracy data appear in Table 1. Analysis of variance revealed a reliable effect of orientation $\left[F(2,22)=3.70, M S_{\mathrm{e}}=.039\right]$. A Fisher's test (lsd $=.166)$ indicated that performance in the $\mathbf{R}$ condition was reliably lower than in the I condition. No other pairwise comparisons differed reliably. Separate $t$ tests showed that the I and C orientations were identified correctly at reliably better than chance levels 
Table 2

Median Confidence Ratings as a Function of Instruction Condition (Experiment 1), Study Orientation, and Orientation Selected at Test

\begin{tabular}{|c|c|c|c|}
\hline \multirow{2}{*}{$\begin{array}{c}\text { Study } \\
\text { Orientation }\end{array}$} & \multicolumn{3}{|c|}{ Orientation Selected at Test } \\
\hline & I & $\mathbf{R}$ & $\mathrm{C}$ \\
\hline \multicolumn{4}{|c|}{ Experiment 1: Incidental Instruction } \\
\hline I & $\begin{array}{l}3.81 \\
(12)\end{array}$ & $\begin{array}{r}2.00 \\
(5)\end{array}$ & $\begin{array}{r}2.73 \\
(10)\end{array}$ \\
\hline $\mathbf{R}$ & $\begin{array}{r}2.89 \\
(8)\end{array}$ & $\begin{array}{l}3.38 \\
(12)\end{array}$ & $\begin{array}{r}2.63 \\
(8)\end{array}$ \\
\hline $\mathrm{C}$ & $\begin{array}{r}2.67 \\
(9)\end{array}$ & $\begin{array}{r}2.21 \\
(7)\end{array}$ & $\begin{array}{l}3.51 \\
(12)\end{array}$ \\
\hline \multicolumn{4}{|c|}{ Experiment 1 : Intentional Instruction } \\
\hline I & $\begin{array}{r}4.00 \\
(12)\end{array}$ & $\begin{array}{r}3.15 \\
(5)\end{array}$ & $\begin{array}{r}3.60 \\
(8)\end{array}$ \\
\hline $\mathbf{R}$ & $\begin{array}{r}3.28 \\
(9)\end{array}$ & $\begin{array}{l}4.24 \\
(12)\end{array}$ & $\begin{array}{r}3.26 \\
(7)\end{array}$ \\
\hline $\mathrm{C}$ & $\begin{array}{l}3.00 \\
(11)\end{array}$ & $\begin{array}{r}3.31 \\
(9)\end{array}$ & $\begin{array}{l}4.01 \\
(12)\end{array}$ \\
\hline \multicolumn{4}{|c|}{ Experiment 2} \\
\hline I & $\begin{array}{l}4.01 \\
(18)\end{array}$ & $\begin{array}{r}2.83 \\
(12)\end{array}$ & $\begin{array}{l}2.97 \\
(12)\end{array}$ \\
\hline $\mathbf{R}$ & $\begin{array}{l}2.97 \\
(12)\end{array}$ & $\begin{array}{r}4.13 \\
(18)\end{array}$ & $\begin{array}{r}3.02 \\
(14)\end{array}$ \\
\hline $\mathrm{C}$ & $\begin{array}{l}2.50 \\
(15)\end{array}$ & $\begin{array}{l}3.28 \\
(13)\end{array}$ & $\begin{array}{l}3.80 \\
(18)\end{array}$ \\
\hline \multicolumn{4}{|c|}{ Experiment 3} \\
\hline I & $\begin{array}{l}4.03 \\
(24)\end{array}$ & $\begin{array}{r}2.96 \\
(13)\end{array}$ & $\begin{array}{l}3.02 \\
(16)\end{array}$ \\
\hline $\mathbf{R}$ & $\begin{array}{l}2.91 \\
(18)\end{array}$ & $\begin{array}{r}3.94 \\
(24)\end{array}$ & $\begin{array}{l}3.40 \\
(20)\end{array}$ \\
\hline $\mathrm{C}$ & $\begin{array}{l}3.27 \\
(20)\end{array}$ & $\begin{array}{r}2.86 \\
(20)\end{array}$ & $\begin{array}{l}3.73 \\
(24)\end{array}$ \\
\hline
\end{tabular}

Note $-\mathrm{I}=$ inverted, $\mathrm{R}=$ reversed, $\mathrm{C}=$ combined (see text). Numbers in parentheses indicate the number of subjects providing at least one score per cell.

$[t \mathrm{~s}(11)=6.38$ and 4.53 , respectively $]$, but the $\mathrm{R}$ orientation was not $(t<1)$. Analysis of confidence ratings for correct responses yielded no effect of orientation $[F(2,22)$ $\left.=1.82, M S_{\mathrm{e}}=1.16\right]$. The confidence rating data appear in Table 2.

\section{Discussion}

The data from Experiment 2 demonstrate that subjects can retain detailed graphemic information over a $48-\mathrm{h}$ delay. The primary evidence for this is the above-chance recognition performance in the $\mathrm{I}$ and $\mathrm{C}$ orientations. A comparison across Experiments 1 and 2, which differed primarily in terms of the delay between the initial reading phase and the recognition test, reveals a substantial drop in recognition accuracy (from .682 to .509). Although the shape of the forgetting function is of course undetermined with only two data points, it seems unlikely that performance on this type of recognition test would be better than chance over more than a few additional days. Masson's (1984) finding that explicit memory for perceptual details of a sentence declined to approximately chance after a 7-day delay is consistent with this hypothesis. Kolers's (1976) observation that sentences are recognizable after more than a year is most likely due to factors other than memory for the graphemic features.

\section{EXPERIMENT 3}

Although it is undoubtedly true that subjects do perform extensive graphemic processing of spatially transformed sentences, it is also possible that the meaningfulness of these materials may attenuate the amount of graphemic processing that is carried out. Thus, the interactive nature of semantic and graphemic processing with such stimuli (Graf \& Levy, 1984) may permit subjects to eliminate some of the graphemic processing that would otherwise be necessary if the semantic information were not available. This suggests that performance on the recognition test for spatial orientation might be enhanced if semantic processing of the stimuli were reduced. This hypothesis was addressed in Experiment 3 by presenting the subjects with anomalous sentences in different spatial transformations. Although the words themselves were obviously meaningful, the normal constraints due to interword relationships and syntax were eliminated, thereby offsetting some of the semantic processing that subjects were able to use in reading meaningful sentences.

\section{Method}

Subjects. Twenty-four subjects were tested individually. The data from 12 additional subjects were discarded as a result of making too many reading errors in one or more cells of the design. Since this attrition rate was comparatively high, all analyses were conducted both on the original 24 subjects and on the final group of 24 . The analyses revealed the same outcomes in terms of statistically reliable differences, although the overall reading times for the original 24 subjects were somewhat longer than those for the final set of 24 subjects. Recognition performance was largely unaffected.

Materials. The procedure used to create the anomalous sentences was as follows. First, a set of 64 meaningful, unrelated sentences was created; each sentence was 15-22 words long. The content words in each sentence were then randomly exchanged with content words from other sentences. The restrictions on this procedure were that no two content words from one sentence could be reassigned to the same sentence and that meaningful relationships among content words in the newly created sentences were to be minimized. Finally, all words in the newly created sentences were randomly ordered, with the restriction that the same word not appear in consecutive positions (e.g., the, of, to).

The sentences were presented in three different orders. Within each order, each sentence appeared equally often in each of the three orientations.

Procedure. In all other regards, the procedure was identical to that used for the incidental learning subjects of Experiment 1

\section{Results}

Reading times. Analysis of median reading times revealed a reliable effect of orientation $[F(2,46)=63.05$, $\left.M S_{\mathrm{e}}=99.96\right]$. A Fisher's test $(\mathrm{lsd}=5.83)$ indicated that all pairwise comparisons differed reliably. The median reading times were $47.60,38.52$, and $16.12 \mathrm{sec}$ for the $\mathrm{I}, \mathrm{R}$, and $\mathrm{C}$ orientations, respectively. As expected, this pattern parallels that found with meaningful sentences. The mean error rates were $14.35 \%, 11.11 \%$, and $1.85 \%$ for the $\mathrm{I}, \mathrm{R}$, and $\mathrm{C}$ orientations, respectively.

Recognition test. The recognition accuracy data appear in Table 1. Analysis of variance revealed a reliable effect of orientation $\left[F(2,46)=4.97, M S_{\mathrm{e}}=.049\right]$. A 
Fisher's test (lsd $=.129)$ indicated that performance in the I condition was reliably better than that in the $\mathrm{R}$ and $\mathrm{C}$ conditions, which did not differ. Separate $t$ tests showed that all three orientations were identified at reliably better than chance levels $[t \mathrm{~s}=10.43,5.28$, and 5.11 for the I, R, and C conditions, respectively]. Analysis of confidence ratings for correct responses on the recognition test revealed no effect of orientation $\left[F(2,46)=1.18, M S_{\mathrm{e}}\right.$ $=0.40]$. These data appear in Table 2 .

\section{Discussion}

Once again, recognition of spatial orientation was reliably better than chance. However, a comparison of performance in Experiments 1 and 3 provides no evidence that eliminating semantic and syntactic constraints enhances memory for graphemic details. One interpretation of this outcome is that elaboration of graphemic processing augments the retention of this information to some upper limit, at least as defined on the recognition test, but further elaboration has no additional effect. This conclusion is consistent with the finding, from all three experiments, that reading time is not a clear predictor of recognition accuracy. Specifically, much faster reading of $\mathrm{C}$ sentences did not result in poorer recognition of the orientation of these sentences relative to $\mathrm{R}$ sentences, for which reading times were at least double. This does not rule out the possibility that other manipulations might result in a further increase in memorability of orientation or of other nonsemantic details, but meaningfulness of the materials does not appear to have a strong effect within the present experimental conditions.

\section{GENERAL DISCUSSION}

Other researchers have concluded that graphemic information (Kolers, 1979) or both semantic and graphemic information (Masson, 1986; Masson \& Sala, 1978) are retained from the reading of spatially transformed text. Although earlier reports have provided convincing evidence that semantic information is a key component of the reading task (Graf, 1981; Horton, 1985; Masson \& Sala, 1978), the present experiments provide the first unequivocal evidence that graphemic details of spatially transformed text are accessible on an explicit memory test. The data from all previous studies are limited in their interpretability by problems of design or analysis.

The present data are entirely consistent with findings from a variety of other research paradigms. Indeed, an important role has been assigned to nonsemantic features in a long list of tasks and phenomena, the most recent of which include the spacing effect (e.g., Dellarosa \& Bourne, 1985), perceptual identification tasks (e.g., Jacoby, 1983; Jacoby \& Hayman, 1987), some recognition tests (Johnston et al., 1985), and some generation phenomena (Glisky \& Rabinowitz, 1985; Slamecka \& Fevreiski, 1983).

Taken together, the present results, along with those of earlier studies, suggest the straightforward conclusion that finding evidence of memory for semantic or nonsemantic information from prior reading of spatially transformed text is determined largely by the measures used. When only nonsemantic features are usable, as in the present experiments, subjects demonstrate excellent memory for such features. When either semantic or nonsemantic features or both are usable, subjects show a strong tendency to utilize the semantic features (e.g., Graf \& Levy, 1984; Horton, 1985; Masson \& Sala, 1978). These conclusions are consistent with the more general view that the major determinant of memory performance is the interaction of processing occurring at encoding and retrieval (e.g., Jacoby \& Brooks, 1984; Johnson, 1983; Kolers \& Roediger, 1984; Morris, Bransford, \& Franks, 1977).

One apparent empirical discrepancy in this literature on the reading of spatially transformed text may also be interpreted in light of these conclusions. Horton (1985), Masson and Sala (1978), and Tardif and Craik (1989) found no evidence that memory for specific nonsemantic features affects the rereading-time measure, whereas Masson (1986) found reliable evidence that these nonsemantic features do affect rereading times. In the former studies, subjects were presented with meaningful sentences both at study and at test, thereby allowing for meaningful context processed at study to be completely reinstated at test. By contrast, Masson's (1986) subjects were presented with strings of unrelated words. These materials would be expected to reduce the amount of semantic processing subjects would invoke, and therefore would be expected to yield processing effects due to other types of information, such as nonsemantic information. Thus, these data seem quite consistent with the idea that rereading times are determined by the interaction of processing occurring at study and at test.

\section{REFERENCES}

Aronson, E. (1976). The social animal. San Francisco: W. H. Freeman Bransford, J. D., \& Franks, J. J. (1971). The abstraction of linguistic ideas. Cognitive Psychology, 2, 331-350.

Craik, F. I. M., \& Tulving, E. (1975). Depth of processing and the retention of words in episodic memory. Journal of Experimental Psychology: General, 104, 268-294.

Dellarosa, D., Bourne, L. E., JR. (1985). Surface form and the spacing effect. Memory \& Cognition, 13, 529-537.

Frost, N. (1972). Encoding and retrieval in visual memory tasks. Journal of Experimental Psychology, 95, 317-326.

Geiselman, R. E. , \& Bellezza, F. S. (1976). Long-term memory for speaker's voice and source location. Memory \& Cognition, 4, 483-489.

Geiselman, R. E., \& BellezzA, F. S. (1977). Incidental retention of speaker's voice. Memory \& Cognition, 5, 658-665.

Geiselman, R. E., \& BJoRk, R. A. (1980). Primary versus secondary rehearsal in imagined voices: Differential effects on recognition. Cognitive Psychology, 12, 188-205.

Glisky, E. L., \& Rabinowitz, J. C. (1985). Enhancing the generation effect through repetition of operations. Joumal of Experimental Psychology: Learning, Memory, \& Cognition, 11, 193-205.

Graesser, A., II, \& Mandler, G. (1975). Recognition memory for the meaning and surface structure of sentences. Jourmal of Experimental Psychology: Human Learning \& Memory, 1, 238-248.

GraF, P. (1981). Reading and generating normal and transformed text. Canadian Journal of Psychology, 35, 293-308. 
Graf, P., Levy, B. A. (1984). Reading and remembering: Conceptual and perceptual processing involved in reading rotated passages Joumal of Verbal Learning \& Verbal Behavior, 23, 405-424.

HorTon, K. D. (1985). The role of semantic information in reading spatially transformed text. Cognitive Psychology, 17, 66-88.

HuNT, R. R., Eluot, J. M. (1980). The role of nonsemantic information in memory: Orthographic distinctiveness effects on retention. Journal of Experimental Psychology: General, 109, 49-74.

JACOBY, L. L. (1983). Remembering the data: Analyzing interactive processes in reading. Joumal of Verbal Learning \& Verbal Behavior. $22,485-508$.

JACOBY, L. L., \& Brooks, L. R. (1984). Nonanalytic cognition: Memory, perception, and concept learning. In G. H. Bower (Ed.), The psychology of learning and motivation (Vol. 18, pp. 1-47). New York: Academic Press.

Jacoby, L. L., \& Hayman, C. A. G. (1987). Specific visual transfer in word identification. Journal of Experimental Psychology: Learming, Memory, \& Cognition, 13, 456-463.

Johnson, M. K. (1983). A multiple-entry, modular memory system. In G. H. Bower (Ed.), The psychology of learning and motivation (Vol. 17, pp. 81-123). New York: Academic Press.

Johnston, W. A., Dark, V. J., JACOBY, L. L. (1985). Perceptual fluency and recognition judgments. Joumal of Experimental Psychology: Learning, Memory, \& Cognition, 11, 3-11.

Kolers, P. A. (1973). Remembering operations. Memory \& Cognition, 1, 347-355.

Kolers, P. A. (1974). Two kinds of recognition. Canadian Journal of Psychology, 28, 51-61.

Kolers, P. A. (1975a). Memorial consequences of automatized encoding. Joumal of Experimental Psychology: Human Leaming \& Memory, 1, 689-701.

Kolers, P. A. (1975b). Specificity of operations in sentence recognition. Cognitive Psychology, 7, 289-306.

Kolers, P. A. (1976). Reading a year later. Journal of Experimental Psychology: Human Learning \& Memory, 2, 554-565.

Kolers, P. A. (1979). A pattern-analyzing basis of recognition. In L. S Cermak \& F. I. M. Craik (Eds.), Levels of processing in human memory (pp. 363-384). Hillsdale, NJ: Erlbaum.

Kolers, P. A., \& OsTrY, D. J. (1974). Time course of loss of information regarding pattern analyzing operations. Journal of Verbal Learning \& Verbal Behavior, 13, 599-612.

Kolers, P. A., \& Roediger, H. L., III. (1984). Procedures of mind Joumal of Verbal Learning \& Verbal Behavior, 23, 425-449.

MANDLeR, G. (1980). Recognizing: The judgment of previous occurrence. Psychological Review, 87, 252-271.

MAsson, M. E. J. (1984). Memory for the surface structure of sentences: Remembering with and without awareness. Journal of Verbal Learning \& Verbal Behavior, 23, 579-592.
MAsson, M. E. J. (1986). Identification of typographically transformed words: Instance-based skill acquisition. Joumal of Experimental Psychology: Leaming, Memory, \& Cognition, 12, 479-488.

MASSON, M. E. J., SALA, L. S. (1978). Interactive processes in sentence comprehension and recognition. Cognitive Psychology, 10, 244-270.

Morris, C. D., Bransford, J. D., \& Franks, J. J. (1977). Levels of processing versus transfer appropriate processing. Journal of Verbal Learning \& Verbal Behavior, 16, 519-533.

Postman, L. \& Kruesi, E. (1977). The influence of orienting tasks on the encoding and recall of words. Journal of Verbal Learning \& Verbal Behavior, 16, 353-369

Sl.amecka, N. J., \& Fevreiski, J. (1983). The generation effect when generation fails. Journal of Verbal Learning \& Verbal Behavior, 22, $153-163$

Tardif, T., \& Craik, F. I. M. (1989). Reading a week later: Perceptual and conceptual factors. Joumal of Memory \& Language, 28, $107-125$.

YeKovich, F. R., Thorndyke, P. W. (1981). An evaluation of alternative functional models of narrative schemata. Journal of Verbal Learning \& Verbal Behavior, 20, 454-469.

\section{NOTE}

1. Some confusion is introduced by Kolers's descriptions of exactly what each of these $d^{\prime}$ values actually measures. At one point, Kolers and Ostry $(1974$, p. 601$)$ stated that " $d$ '(old) is a measure primarily of the graphemic component," but they subsequently suggested that it "assess[es] a more pictorial memory of the graphemes" (p. 601), a view consistent with other summaries (e.g., Kolers, 1979, p. 367). Assuming that these two components, graphemic and pictorial, are theoretically and empirically separable, it is interesing to note that the former description of $d^{\prime}$ (old) is entirely consistent with the description suggested here.

A problem in the calculation of $d^{\prime}$ (old) may also be noted. Kolers (1974) presented subjects with sentences that appeared in normal (N) inverted (I), or reversed-rotated (rR) orientation at study. These study orientations were factorially combined with the same three orientations on the recognition test. (To illustrate the notation, sentences studied in the $\mathrm{N}, \mathrm{I}$, and $\mathrm{rR}$ orientations and tested in the $\mathrm{N}$ orientation are identified as NN, IN, and rRN, respectively.) Kolers $(1974$, p. 55) noted that, for one of the calculations of $d^{\prime}$ (old), rRN sentences placed in the differen form pile were used as hits, with NN and IN sentences placed in this pile used as false alarms. Obviously, an IN sentence placed in the different form pile is not a false alarm by any criterion.

(Manuscript received October 28, 1987; revision accepted for publication September 20, 1988.) 